\title{
Pulmonary focal fibrosis associated with microscopic arterio-venous fistula manifesting as focal ground-glass opacity on thin-section CT
}

Noriko Sudo ${ }^{1 *}$, Atsushi Nambu1, Takana Yamakawa', Masashi Kawamoto², Shozo Fujino ${ }^{3}$, Masato Watanabe $^{3}$, Kunio Mizuguchi ${ }^{2}$ and Masao Tago ${ }^{1}$

\begin{abstract}
Background: Focal ground-glass opacity (GGO) on thin-section computed tomography (CT) may be seen in atypical adenomatous hyperplasia (AAH), adenocarcinoma in situ that has recently been renamed from bronchioloalveolar carcinoma (BAC) and various benign conditions.

Case presentation: We report a case of pulmonary focal fibrosis associated with microscopic arterio-venous fistula $(\mathrm{AVF})$, which showed a focal area of GGO on thin-section $\mathrm{CT}$. The patient was a 58-year-old woman with a GGO on thin-section $C T$ which had increased in size over the period of 2 years. Slightly dilated vessels and thickened interlobular septa were also noted around the GGO. It was diagnosed preoperatively as adenocarcinoma in situ and a partial lung resection by video-assisted thoracic surgery (VATS) was performed. Pathological examination yielded a diagnosis of focal fibrosis associated with microscopic AVF.

Conclusion: We speculate that the focal fibrosis was produced by a prolonged congestion due to the AVF and that the dilated vessels and thickening of interlobular septa on thin-section CT related to the AVF. Microscopic AVF may be one of the etiologies of focal fibrosis showing focal GGO on thins-section CT. Dilated vessels and thickened interlobular septa around the GGO might offer a clue to the diagnosis of this disease entity. In addition, it should be noted that focal fibrosis may increase in size.
\end{abstract}

Keywords: Ground-glass opacity, Focal fibrosis, Lung, CT, Arterio-venous fistula

\section{Background}

Recently, widespread use of computed tomography (CT) for lung cancer screening allows us to detect many faint peripheral pulmonary lesions. Radiologists have come to encounter focal ground-glass opacity (GGO) more frequently than before. Persistent focal GGO is most commonly seen in atypical adenomatous hyperplasia (AAH) and adenocarcinoma in situ that has recently been renamed from bronchioloalveolar carcinoma (BAC) in the revised classification [1]. Focal GGO may also be seen in various benign conditions, including focal fibrosis, focal inflammation, or hemorrhage [2,3]. Focal

\footnotetext{
* Correspondence: nonnon07@hotmail.co.jp

'Department of Radiology, Teikyo University Mizonokuchi Hospital, 3-8-3

Mizonokuchi Takatsu-ku, Kawasaki City, Kanagawa 213-8507, Japan

Full list of author information is available at the end of the article
}

fibrosis accounts for approximately $15 \%$ of focal pure GGO [4]. However its etiology is still unknown.

Herein, we present a case of focal fibrosis associated with microscopic arterio-venous fistula (AVF) manifesting as focal GGO mimicking adenocarcinoma in situ on chest CT, which suggested a pathogenesis of focal fibrosis.

\section{Case presentation}

A 58-year-old woman was referred to our hospital for detailed evaluation of focal GGO that had been discovered by a screening CT two years before. Thirteen years earlier, the patient had undergone a partial left lung resection for pulmonary hamartoma. Biochemical tests and tumor markers for lung cancer on admission were within the normal ranges. Her physical condition was good with no clinical symptoms. She had never smoked.

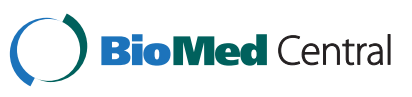




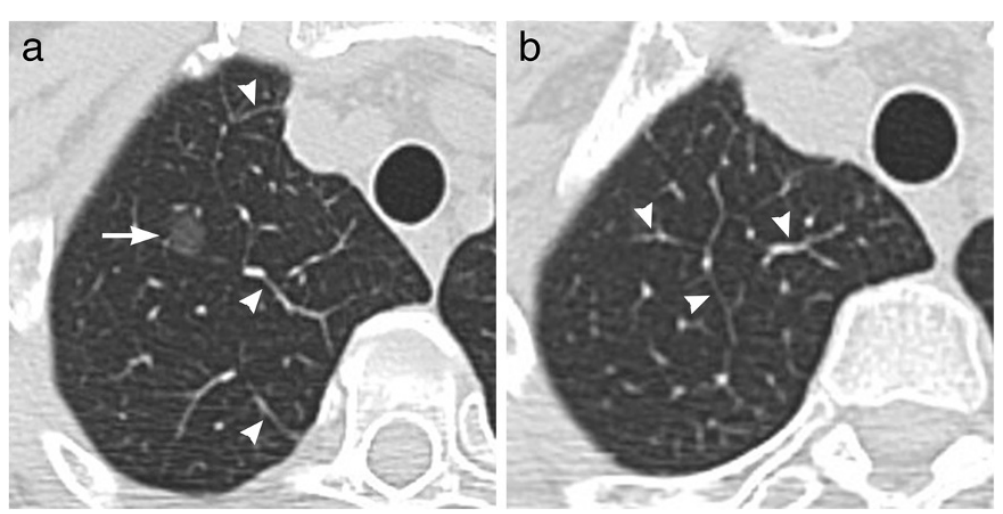

Figure 1 Chest thin-section CT on admission to our hospital. (a) A thin-section CT at the level of right upper lobe shows a 10-mm round, well-defined GGO nodule (arrow). There are slightly dilated veins or thickening of the interlobular septa (arrow heads). (b) Similar findings are seen also on the slice just above the focal GGO (arrow heads).

Chest thin-section CT scan showed a 10-mm pure GGO with a well-defined and smooth margin in the right upper lobe (Figure 1a). In addition, slightly dilated veins and thickening of interlobular septa were seen around the focal GGO (Figure 1a, b).

The focal GGO was identifiable also on the previous $\mathrm{CT}$ for lung cancer screening when viewed retrospectively (Figure 2). It had increased in size for 2 years. The lesion was not identified on chest radiograph. Adenocarcinoma in situ was considered likely due to the presence of an interval increase of the GGO. The lesion was resected by video-assisted thoracic surgery (VATS). It was unidentifiable during the surgery. Histopathological

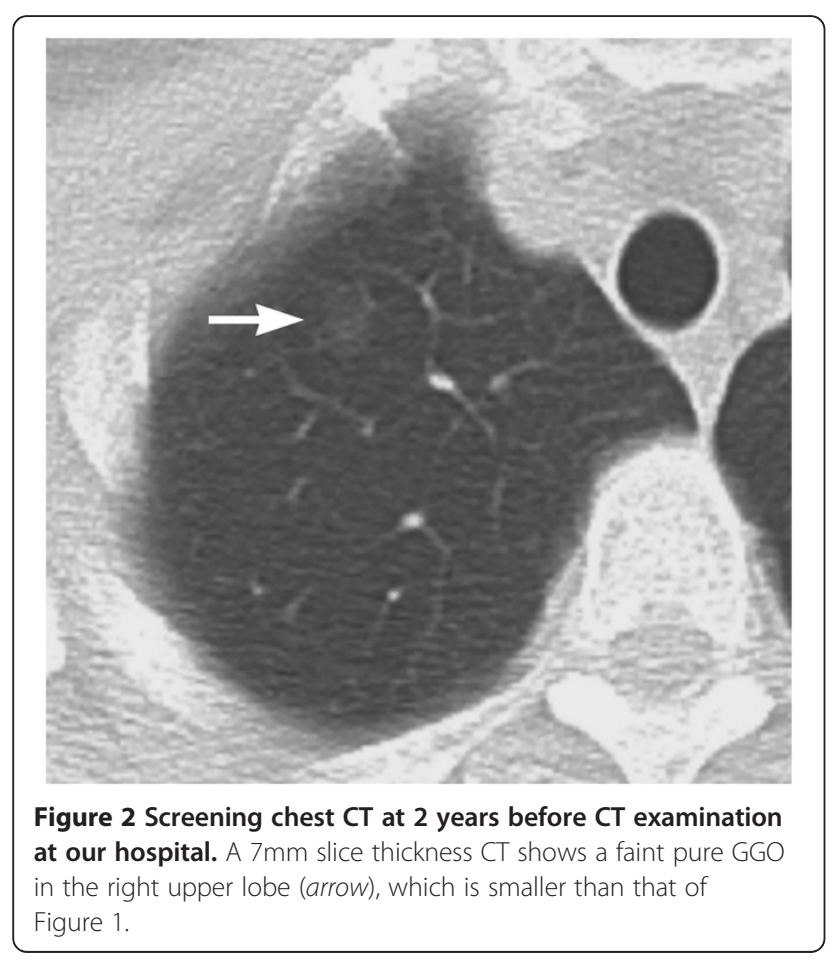

examination of the resected lung tissue revealed no evidence of malignancy but abnormally dilated arterioles, venules and lymph ducts with an area of peripheral focal fibrosis consisting of congestion, fibrotic septal thickening with preservation of the intraalveolar airspaces (Figure $3 \mathrm{a}-\mathrm{c}$ ). This focal fibrosis was considered to correspond to the focal GGO on thin-section CT. The dilated arterioles had thickened walls and were directly communicating with the venules (Figure 3c). These histopathological findings were similar to those of arteriovenous malformation (AVM). However, the present case showed no significant evidence of nidus and it was unknown whether these vascular abnormalities were congenital or not. Therefore, we regarded it as microscopic AVF.

\section{Discussion}

Pure GGO that is persistently (either no change or an increase in diameter for $\geqq 1$ month) present on serial thin-section CT scans suggests the possibility of AAH, adenocarcinoma in situ, pulmonary lymphoproliferative disorder, or organizing pneumonia/focal fibrosis [5]. The most common diseases showing persistent focal GGO are AAH and adenocarcinoma in situ. Focal fibrosis is a relatively rare disease entity constituting $15-21 \%$ of persistent focal GGO [3,4].

The pathogenesis of focal fibrosis is still not well understood. It may represent a focal tissue response to local lung injury due to infection or radiation, drug, or physical pressure [6]. In our case, microscopic AVF coexisted with the focal fibrosis. We speculate that prolonged localized congestion caused by AVF lead to focal tissue fibrosis. Of note in our case is that the focal fibrosis had increased in size during the time interval between the two CT examinations. As congestion associated with AVF is considered a chronic pathophysiological process, the fibrosis may have gradually extended. 


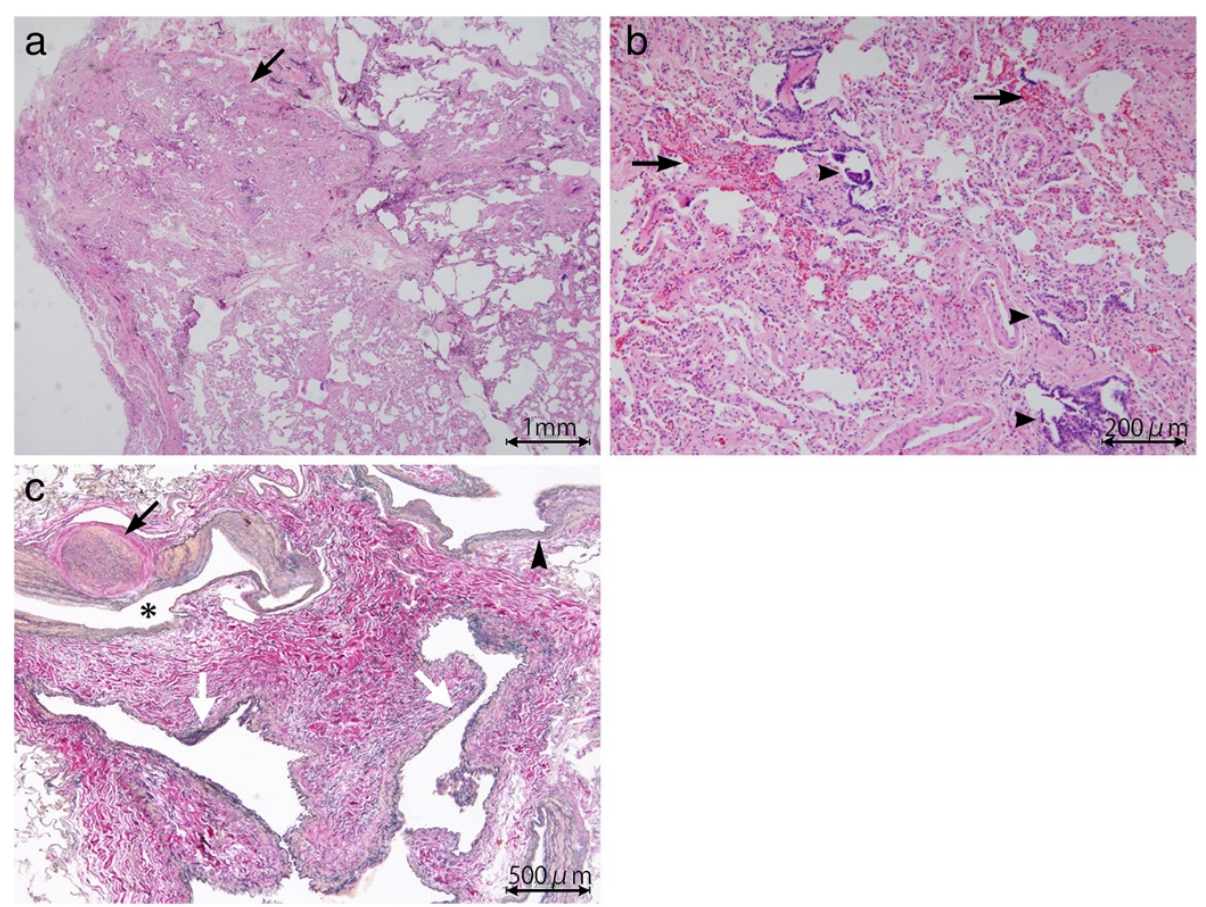

Figure 3 Histopathology of the resected lung tissue. (a) Photomicrograph (hematoxylin-eosin stain; original magnification, $\times 2$ ) shows a focal area of alveolar wall thickening with preservation of the intraalveolar airspaces, consistent with the focal GGO on thin-section CT (arrow). Partial collapse of this lesion is due to an artifact during the process of specimen production. (b) Photomicrograph (hematoxylin-eosin stain; original magnification, $\times 10$ ) shows alveolar wall thickening with fibrosis, and congestion(arrows). Alveolar bronchiolizations are also seen (arrowheads). (c) Photomicrograph (elastica Masson stain; original magnification, $\times 4$ ) near the focal fibrosis shows dilated arterioles (asterisk) with markedly thickened walls, one of which have resulted in luminal obliteration (black arrow), as well as dilated venules (white arrows). A transition from arteriolar wall to venular one is seen, suggesting a direct communication between them (arrow head).

Focal fibrosis has been described as sharply demarcated nodular GGO with a maximal diameter of less than $2 \mathrm{~cm}$ on thin-section CT [4]. Solid components which pathologically correspond to compact fibrotic foci or alveolar collapse may be present. These imaging features are shared by both focal fibrosis and AAH/ adenocarcinoma in situ. Therefore, the differentiation between these lesions by CT images is difficult. One possible discriminating feature may be that focal fibrosis does not increase in size over a considerable period of time [6]. However, our case showed an interval growth over a few years.

F-18 fluorodeoxyglucose positron emission tomography $\left({ }^{18}\right.$ F-FDG PET) has increasingly been used in the evaluation of lung cancer. It is also useful for differentiating between malignant and benign nodules. However, as for focal GGO, it has been shown that ${ }^{18}$ F-FDG PET has limited value in the evaluation of focal GGOs for determining nodule malignancy and staging in comparison with solid nodules. Chun et al. [7] reported that in pure GGOs, both inflammation and malignancy showed an SUV less than 1.0 and did not show a statistically significant difference. Therefore, ${ }^{18}$ F-FDG PET is considered to contribute little to the differentiation between adenocarcinoma in situ and focal fibrosis.

A well-known imaging feature of pulmonary AVF is dilatation of the feeding artery and draining vein with a nodular area where these vessels join. We couldn't see such a nodular area in our case probably because the AVF was microscopic and thus was too small to see on CT. However, slightly dilated veins and thickened interlobular septa were demonstrated around the focal GGO on thin-section CT. We think that these findings related to the AVF and may be a clue to the differentiation between this disease entity and AAH/adenocarcinoma in situ.

\section{Conclusions}

In summary, we presented a rare case of focal fibrosis associated with microscopic AVF that showed focal GGO on thin-section CT. To our knowledge, this is the first report of this disease entity. Although the CT findings are similar to those of AAH/adenocarcinoma in situ, dilated vessels and thickened interlobular septa around the GGO might offer a clue to the differential 
diagnosis. Additionally, it should be noted that focal fibrosis may increase in size.

\section{Consent}

Written informed consent was obtained from the patient for publication of this Case report and any accompanying images. A copy of the written consent is available for review by the Editor of this journal.

\section{Abbreviations}

AVF: Arterio-venous fistula; GGO: Ground-glass opacity; CT: Computed tomography; BAC: Bronchioalveolar carcinoma; VATS: Video-assisted thoracic surgery; AAH: Adenomatous hyperplasia; AVM: Arteriovenous malformation;

${ }^{18}$ F-FDG PET: F-18 fluorodeoxyglucose positron emission tomography.

\section{Competing interests}

We have no competing interests.

\section{Authors' contributions}

NS drafted the initial manuscript and modified it in reference to the other authors' opinion. AN, TY and MT edited the manuscript. MK, SF, MW and KM were involved in diagnostics and treatment of the patient and provided advice to me in each of their expertise areas. All authors read and approved the final manuscript.

\section{Author details}

1Department of Radiology, Teikyo University Mizonokuchi Hospital, 3-8-3

Mizonokuchi Takatsu-ku, Kawasaki City, Kanagawa 213-8507, Japan.

${ }^{2}$ Department of Clinical Pathology, Teikyo University Mizonokuchi Hospital,

3-8-3 Mizonokuchi Takatsu-ku, Kawasaki City, Kanagawa 213-8507, Japan.

${ }^{3}$ Department of Surgery, Teikyo University Mizonokuchi Hospital, 3-8-3

Mizonokuchi Takatsu-ku, Kawasaki City, Kanagawa 213-8507, Japan.

Received: 2 November 2012 Accepted: 10 January 2013

Published: 14 January 2013

\section{References}

1. Travis WD, Brambilla E, Noguchi M, Nicholson AG, Geisinger KR, Yatabe $Y$, Beer DG, Powell CA, Riely GJ, Van Schil PE, Garg K, Austin JH, Asamura H, Rusch W, Hirsch FR, Scagliotti G, Mitsudomi T, Huber RM, Ishikawa Y, Jett J, Sanchez-Cespedes M, Sculier JP, Takahashi T, Tsuboi M, Vansteenkiste J, Wistuba I, Yang PC, Aberle D, Brambilla C, Flieder D, et al: International association for the study of lung cancer/american thoracic society/ european respiratory society international multidisciplinary classification of lung adenocarcinoma. J Thorac Oncol 2011, 6:244-285.

2. Chang MP, Jin MG, Hyun JL, Chang HL, Eun JC, Jung-Gi I: Nodular groundglass opacity at thin-section CT: histopathologic correlation and evaluation of change at follow-up. RadioGraphics 2007, 27:391-408.

3. Collins J, Stern EJ: Ground-glass opacity at CT: The ABCs. AJR Am J Roentgenol 1997, 169:355-367.

4. Nakajima R, Yokose T, Kakinuma R, Nagai K, Nishiwaki Y, Ochiai A: Localized pure ground-glass opacity on high-resolution $\mathrm{CT}$ : histologic characteristics. J Comput Assist Tomogr 2002, 26:323-329.

5. Kim HY, Shim YM, Lee KS, Han J, Yi CA, Kim YK: Persistent pulmonary nodular ground-glass opacity at thin-section CT: histopathologic histopathologic comparisons. Radiology 2007, 245:267-275.

6. Chang MP, Jin MG, Hyun JL, Doo HC, Eun JC, Jung-Gi I: Focal interstitial fibrosis manifesting as nodular ground-glass opacity: thin-section CT findings. Eur Radiol 2007, 17:2325-2331.

7. Chun EJ, Lee HJ, Kang WJ, Kim KG, Goo JM, Park CM, Lee CH: Differentiation between malignancy and inflammation in pulmonary ground-glass nodules: the feasibility of integrated ${ }^{18} \mathrm{~F}-\mathrm{FDG}$ PET/CT. Lung Cancer 2009, 65:180-186.

doi:10.1186/1471-2466-13-3

Cite this article as: Sudo et al.: Pulmonary focal fibrosis associated with microscopic arterio-venous fistula manifesting as focal ground-glass opacity on thin-section CT. BMC Pulmonary Medicine 2013 13:3.

\section{Submit your next manuscript to BioMed Central and take full advantage of:}

- Convenient online submission

- Thorough peer review

- No space constraints or color figure charges

- Immediate publication on acceptance

- Inclusion in PubMed, CAS, Scopus and Google Scholar

- Research which is freely available for redistribution 